Chirurg 2014 · 85:914

DOI 10.1007/s00104-014-2875-y

Online publiziert: 13. September 2014

c) Springer-Verlag Berlin Heidelberg 2014

H. Dralle $\cdot M$. Elwerr

Universitätsklinik für Allgemein-, Viszeral- und Gefäßchirurgie, Medizinische Fakultät,

Universitätsklinikum Halle, Halle (Saale)

\title{
Adrenalektomie bei Metastasen
}

\section{Ergebnisse}

In die Studie eingeschlossen wurden 317 zwischen 1999 und 2011 wegen NNM adrenalektomierte Patienten. Demographische Daten: $23 \%$ der Metastasen traten synchron auf, isolierte Metastasen in $74 \%$, laparoskopische Operationen erfolgten in $46 \%$, die Resektion war beschränkt auf die Nebenniere in 73\%, R1/R2-Resektionen lagen in $8 \% / 6 \%$ vor. Die mittlere Tumorgröße betrug $6 \mathrm{~cm}$. Über $50 \%$ der Patienten erhielten eine adjuvante Therapie. Die wichtigsten Verlaufsergebnisse waren folgende:

- Das mediane Gesamtüberleben lag bei 29 Monaten, die 5-Jahres-Überlebensrate bei $35 \%$. Hauptursachen für Versterben waren Tumorprogression (78\%), nur selten Hospitalletalität (3\%). Die Art des Primärtumors (Nierenkarzinome > Kolorektalkarzinome $>$ nichtkleinzellige Lungenkarzinome) und die Chronizität (metachron > synchron) hatten einen signifikanten Einfluss auf das Überleben.

- Multivariat wurden folgende Faktoren als ungünstige Voraussetzungen für Überleben identifziert: keine Chemotherapie und keine Resektion des Primärtumors, keine radikale Adrenalektomie der Metastase.

- Bivariat waren neben metachroner Metastasierung in die Nebenniere und fehlender extraadrenaler Invasion auch das laparoskopische Vorgehen von Vorteil für das Langzeitüberleben.

\section{Diskussion und Fazit}

Die große Zahl der in der vorliegenden Untersuchung eingeschlossenen $\mathrm{Pa}$ tienten mit NNM bestätigt die Ergebnisse früherer Untersuchungen mit klei- nerer Fallzahl. Prospektiv randomisierte Studien wird es wegen der Seltenheit von NNM auch in Zukunft kaum geben. Die beste Operationsindikation sind metachrone, isolierte Metastasen von Nierenzellkarzinomen, die radikal reseziert werden können. Metastasen von nichtkleinzelligen Lungenkarzinomen sind die häufigsten Nebennierenmetastasen, aber zugleich die prognostisch ungünstigsten. Das in der vorliegenden Studie in der Hälfte der Fälle erfolgte laparoskopische Vorgehen war gegenüber dem offenen Vorgehen eher von Vorteil für das Outcome, solange keine die Nebenniere überschreitende Organinvasion vorlag. Bei allen Limitationen, die mit einer retrospektiven Studie der vorliegenden Art verbunden sind, lassen die Ergebnisse den Schluss zu, dass die Indikation zur Adrenalektomie bei solitärer Nebennierenmetastasierung in der Regel gegeben ist, und dass bei fehlender Organüberschreitung und vertretbarer Größe ein endoskopisches Vorgehen zu bevorzugen ist.

\section{Korrespondenzadresse}

\section{Prof. Dr. Dr. h.c. H. Dralle}

Universitätsklinik für Allgemein-, Viszeralund Gefäßchirurgie, Medizinische Fakultät, Universitätsklinikum Halle, Ernst-Grube-Str. 40, 06097 Halle (Saale) henning.dralle@uk-halle.de

Interessenkonflikt. H. Dralle und M. Elwerr geben an, dass kein Interessenkonflikt besteht.

\section{Literatur}

1. Creamer J, Matthews BD (2013) Laparoscopic adrenalectomy for cancer. Surg Oncol Clin N Am 22:111-124 